\title{
Hautberuhigendes Reinigungserlebnis für Gesicht und Körper
}

Die abgestimmte Reinigung ist eine wichtige Basis, um die Haut im Gleichgewicht zu halten und diese auf die wei-

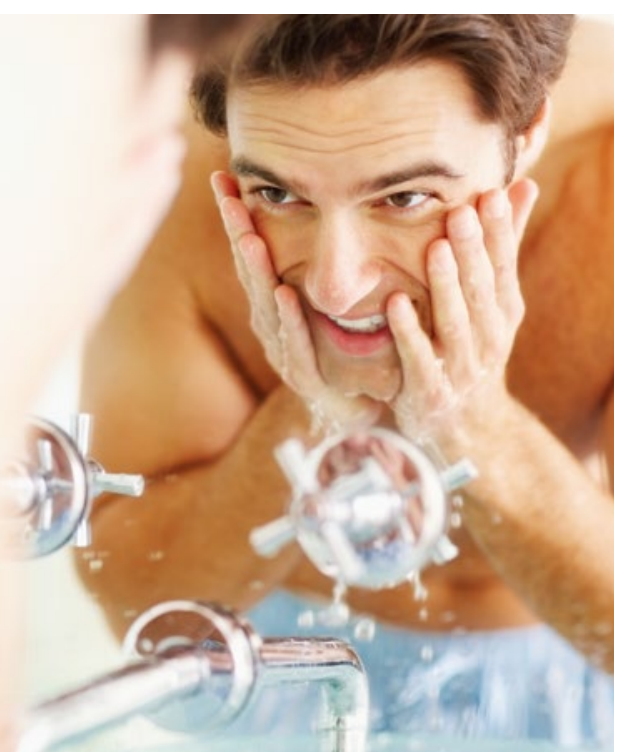

Schonende Reinigungsprodukte schützen gerade bei sensibler Haut die Hautbarriere. tere hauttypgerechte Gesichtspflege vorzubereiten. Besonders für die sensible, $\mathrm{zu}$ Rötungen und Juckreiz neigende Haut sind schonende Reinigungsprodukte, die die Haut nicht unnötig reizen oder zusätzlich belasten, zu empfehlen. Der anti-irritative DERMASENCE BarrioPro Reinigungsschaum reinigt und beruhigt sensible Haut, die zu Rötungen und Juckreiz neigt. Die irritierte Hautbarriere im Gesicht und am Körper wird geschützt und gestärkt. Färberwaidextrakt wirkt antiinflammatorisch und antimykotisch, bringt die Mikroflora ins Gleichgewicht und optimiert so die Widerstandskraft der Haut. Der milde Reinigungsschaum ist auch zur Rasur empfindlicher Haut geeignet sowie als schonende Reinigung bei perioraler Dermatitis und bei diabetischen Hautproblemen.

\section{Spezialpflege mit dem Plus an Feuchtigkeit}

Nicht nur der Winter ist eine belastende Saison für die Haut, auch im Frühling leiden noch viele Betroffene an trockener und schuppiger Haut. Um zu verhindern, dass sich diese Erscheinungen zu einem ganzjährigen Hautproblem entwickeln, sollte rechtzeitig die passende Spezialpflege eingesetzt werden. Besonders wichtig ist es, den erhöhten transepidermalen Wasserverlust (TEWL) der Hornschicht wieder in die richtige Balance $\mathrm{zu}$ bringen und die natürliche Barrierefunktion der Haut zu stabilisieren.

Die DERMASENCE Adtop plus ist speziell auf diese Bedürfnisse abgestimmt und somit die ideale Pflege für sehr trockene, schuppige und feuchtigkeitsarme Haut. Die Creme basiert auf den positiven Eigenschaften der DERMASENCE Adtop Creme. Mit dem plus an Urea (10\%) und dessen hydratisierenden Effekt steigt die Hautfeuchtigkeit und Spannungsgefühle werden gemindert.

Bei regelmäßiger Anwendung werden raue, schuppige Hautstellen weich und geschmeidig, ohne dass ein Fettfilm entsteht. Die Creme ist auch ideal bei belasteten Händen und Füßen sowie im Rahmen der Intervalltherapie bei $\mathrm{zu}$ Neurodermitis neigender Haut geeignet. Die Haut fühlt sich geschmeidiger an und wird zusätzlich optimal gepflegt.

Nach Informationen von P\&M Cosmetics

\section{Hyaluronidase unterstützt die Lokalanästhesie}

Die bovine Hyaluronidase (Hylase ${ }^{\circledast}$ „Dessau“) ist als Zusatz zur Infiltrationsanästhesie zugelassen. Gegenüber Placebo vergrößert das Adjuvans die anästhesierte Hautfläche um das 1,4-Fache und der Bereich ist 10 Minuten schneller betäubt. Dabei ist der sogenannte Spreading-Effekt des Enzyms reversibel. „Degradierte körpereigene Hyaluronsäure wird umgehend regeneriert, es entstehen keine negativen Nettoeffekte unter anderem auf das Volumen“, erklärte PD Dr. med. Peter Arne Gerber, Dermatologe am Universitätsklinikum Düsseldorf.

Bei Blepharoplastiken ist der Enzymeinsatz bereits Standard. Hier ermögliche das Adjuvans auch bei subkutaner
Applikation eine Tiefenwirkung bis unter das Septum orbitale, wie Prof. Dr.medic (RO) Alina Fratila, JungbrunnenKlinik, Bonn, berichtete. Bei Eingriffen wie der Liposuktion ist die Hyaluronidasezugabe Bestandteil der Tumeszenslokalanästhesie (TLA). Da sich dadurch die TLA-Lösung reduzieren lasse, blieben im subkutane Fettgewebe extreme Dehnungen und -rupturen weitgehend aus, so Fratila.

Auch bei der lasergestützten Cellulitebehandlung lässt sich der SpreadingEffekt nutzen, um die zu behandelnden Areale besser und mit weniger TLALösung zu infiltrieren. Zudem werde die gewünschte Temperatur der Laser- erwärmung des Gewebes schneller und besser erreicht, erklärte die Dermatologin und Spezialistin für ästhetische Lasermedizin.

Der medizinisch-ästhetische Einsatz von HA-Fillern kann zu Komplikationen führen, die mit Hyaluronidase wieder beseitigt bzw. reduziert werden können. Dazu erklärte Gerber: „Bei zeitnaher Applikation, innerhalb von 4 Stunden nach HA-Filler-Injektion, reduziert Hyaluronidase vaskuläre Komplikationen signifikant“. Auf die Wundheilung hat Hyaluronidase keinen negativen Einfluss, wie In-vitro-Untersuchungen zeigten.

Matthias Manych

Pressekonferenz „Hyaluronidase in der ästhetischen Dermatologie: Einzigartiger Wirkmechanismus als Schlüssel für vielseitige Anwendungsmöglichkeiten" im Rahmen der 49. Tagung der Deutschen Dermatologischen Gesellschaft, Berlin, 27. April 2017; Veranstalter Riemser Pharma 